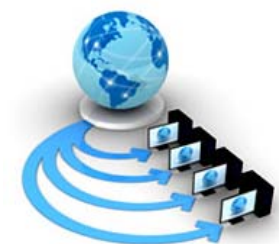

Volume 11, No. 3, May-June 2020

International Journal of Advanced Research in Computer Science

REVIEW AERICLE

Available Online at www.ijarcs.info

\title{
A REVIEW ON FACE RECOGNITION TECHNIQUE USING THE ARTIFICIAL NEURAL NETWORK
}

\author{
Savita Bairagi ${ }^{1}$, Hemant Gupta ${ }^{2}$, Ashok Panwar ${ }^{3}$, Bhavana Panwar ${ }^{4}$, \\ ${ }^{1}$ Research Scholar at Lakshmi Narain College of Technology and Science (RIT), Indore, \\ ${ }^{2} \mathrm{HOD}$ in Computer Science \& Engineering Department at LNCT, Indore, \\ ${ }^{3}$ Technical Officer (C) in ECIL (NPCIL), Tarapur, Mumbai, \\ ${ }^{4}$ Research Scholar as well as Faculty from SVBPHSS, Kasrawad, Khargone, MP
}

\begin{abstract}
Face recognition is one of the classical domains of research and development. Significant contributions on face recognition technique are available in literature. There are various kinds of techniques and methodologies which can be divided in two major categories i.e. feature classification based approach and reconstruction based approaches. In this paper, we are first providing the survey on existing efficient and recent techniques of face recognition. Additionally on the basis of concluded consequences a new model for recognizing the partial face. In this context a new model using LDA based features and BPN (back propagation neural network) based is introduced. Additionally their functional aspects are discussed. Finally the conclusion and future working guidelines are provided.
\end{abstract}

Keywords: face recognition, partial faces, neural network, LDA, survey, machine learning.

\section{I.INTRODUCTION}

Now in these days the machine learning and its techniques are played essential role in various real world applications. These applications are used for pattern recognition, classification, categorization, prediction, motion detection and various others [1]. Additionally the machine learning algorithms also works well with the images for motion detection, activity recognition and others. Among them the face recognition is one of the most essential applications. That is used in wide range of security, surveillance, monitoring and authentication [2].

Face is one of the most essential identities, which is almost different and unique for all the individuals. In a number of applications it is used as a biometric identity and utilized for authentication and access control [3]. But these applications are feasible if the recognition of the faces becomes accurate. The low rate of face recognition can degrade the performance of these applications. The basic issues of these systems are the pose variation, partial capturing of faces, small changes on faces, low resolution images, noisy images and others [4]. Due to these, improvements on the traditional face recognition systems are required. The proposed work is intended to design and develop an accurate face recognition technique that works on different variants of faces and can able to recognize any of the pose obtained by the user data [5].

Therefore in this paper the ORL dataset is used for designing and developing the face recognition system which contains different poses of faces of the same class. In addition of that the LDA (linear discriminant analysis) technique is used for computing face features. The multi-pose based features are used with the back-propagation neural network for training of faces. After training of the neural network the system is able to accept any of the training face pose and can recognize accurately.

This section provides the basic overview of the proposed methodology and the next section provides the essential contributions placed for recognizing the faces. Further the literature summary is offered. Additionally on the basis of concluded facts a new face recognition model is proposed. Finally the conclusion and future directions of the work is provided.

\section{LITERATURE SURVEY}

This section provides the collection of recently available contributions and efforts that are promising for accurately recognizing the faces.

S. S. Farfade et al [6] consider the problem of multi-view face detection. Current state-of-the-art approaches for this task require annotation of facial landmarks, or annotation of poses. They also require training dozens of models to capture faces in all orientations. Authors propose Deep Dense Face Detector (DDFD), a method that does not require pose/landmark annotation and able to detect faces in a wide range of orientations using a single model based on deep CNN. The method has minimal complexity; and not requires additional components. They analyzed scores of the face detector for faces in different orientations and found that 1) method is able to detect faces from different angles, 2) there seems to be a correlation between distribution of positive examples in the training set and scores of the given face detector. The method's performance was improved using better sampling and sophisticated data. Evaluations on benchmark datasets show that 
single-model face detector has better performance compared to the previous methods.

Automatic affect recognition is a challenging task due to the various modalities emotions. Applications can be found in many areas i.e. multimedia retrieval and human computer interaction. $\boldsymbol{P}$. Tzirakis et al [7] propose an emotion recognition system using auditory and visual modalities. To capture the emotions for various styles of speaking, robust features need to be extracted. This purpose, authors utilize a CNN to extract features from the speech, for visual modality a deep residual network (ResNet). Additionally, a machine learning algorithm needs also to be insensitive to outliers while being able to model the context. To tackle this, Long Short-Term Memory networks are utilized. System is then trained in an end-to-end fashion where - by also taking advantage of the correlations of the each of the streams author manage to outperform the traditional approaches based on auditory and visual handcrafted features.

Recently, CNN have demonstrated excellent performance on various tasks, including classification of two-dimensional images. W. Hu et al [8], deep CNN are employed to classify hyper-spectral images. More specifically, the architecture of the classifier contains five layers with weights which are the input layer, the convolutional layer, the max pooling layer, the full connection layer, and the output layer. These layers are implemented on each spectral signature to discriminate against others. Results based on several data sets show that the method can achieve better performance than traditional methods, such as SVM and traditional deep learning- methods.

Z. Yu et al [9] report image based static facial expression recognition method for the Emotion Recognition. Authors focus on the sub-challenge of the SFEW 2.0 dataset, where seeks to classify a set of static images into 7 emotions. The method contains a face detection module based on ensemble of three face detectors, followed by a classification module with the ensemble of multiple deep CNN. Each CNN model is initialized randomly and pre-trained on a larger dataset. The pre-trained models are then fine-tuned on the training set. To combine CNN models, author present two schemes for learning: by minimizing the log likelihood loss, and by minimizing the hinge loss. The method generates result on the FER dataset. It also achieves 55.96\% and $61.29 \%$ respectively.

$X$. Yin et al [10] explores multi-task learning (MTL) for face recognition. They answer the questions of how and why MTL can improve. First, author propose a multi-task CNN for face recognition where identity classification is the main task and pose, illumination, and expression estimations are the side tasks. Second, they develop a dynamic-weighting scheme to automatically assign the loss weight to each side task. Third, they propose a pose-directed multi-task CNN by grouping different poses to learn pose-specific identity features. They propose an energy-based weight analysis method to explore how CNN-based MTL works. They observe that the side tasks serve as regularizations to disentangle the variations from the learnt identity features. Experiments demonstrate the effectiveness of the approach. The approach is also applicable for pose-invariant face recognition and better performance.

The problems facing by blind people fall in the category of navigating through indoor and outdoor consisting of various obstacles and recognition of person in front of them. Identification of objects or person only with perceptive and audio information is difficult. This helps blind people to navigate with the help of a Smartphone, global positioning system (GPS) and a system with ultrasonic sensors. Face recognition can be done using neural learning techniques. The images of friends, relatives are stored in the database. Whenever a person comes in front of the blind user, the application gives the voice aid to the user. Thus system can replace the regular imprecise use of guide dogs and white sticks to help the navigation and face recognition process. P. M. Kumar et al [11] have proposed a novel image recognition and navigation system that provides precise and quick messages in the form of audio to visually challenged people.

The complexity of the human face and the changes due to different effects make it more challenging to design and implement a system for face recognition. M. A. Abuzneid et al [12] presented an approach to improve face recognition using a BPNN and features extraction based on correlation. A key contribution is the generation of a new set called the T-Dataset from the training data set, which is used to train the BPNN. The correlated T-Dataset provides a high distinction layer between the training images, it helps BPNN to converge faster and achieve better accuracy. Additionally authors used LBP histogram to prove that there is potential improvement. They applied five distance measurement algorithms and combine them to obtain the T-Dataset. Authors achieved higher recognition accuracy with less computational cost. Furthermore, author evaluates method on one of the benchmark data set, Labeled Faces, where they produce a competitive face recognition performance.

Thermal face recognition has received increasing attention. The traditional methods for TFR concentrate on the hand-crafted features, which requires more efforts to select and extract features and usually has lower recognition rate. Z. Wu et al [13] present CNN architecture for TFR. It is a new type of ANN that can learn features from the raw data. Results on face database show that CNN achieves higher recognition rate.

S. Kumaar et al [14] present real-time deep neural network architecture for disguised face verification. The model consists of two ANN, first one being CNN that predicts 20 facial keypoints in the image and the second ANN classifies the subject. The accuracies are $67.4 \%$ and $74.8 \%$ for prediction and classification respectively. 
Identification of individual livestock has become a pressing issue as intensification practices continue to be adopted and precise objective measurements are required. Current best practice involves the use of RFID tags which are time-consuming task. To overcome this, non-invasive biometrics is proposed by $\boldsymbol{M}$. $\boldsymbol{F}$. Hansen et al [15]. They test this in a farm, on 10 individual pigs using three techniques: Fisher-faces, the VGG-Face pre-trained face CNN model and own CNN model that train using an artificially augmented data. Results show that accurate pig recognition is possible with accuracy rates of $96.7 \%$ on 1553 images.

\section{LITERATURE SUMMARY}

This section provides the summary of the collected literature. The table 3.1 includes the different aspects of the proposed study.

\begin{tabular}{|c|c|c|}
\hline $\begin{array}{l}\text { Authors and } \\
\text { publication }\end{array}$ & Contribution & Outcomes \\
\hline $\begin{array}{l}2015 \text { ACM, S. S. } \\
\text { Farfade et al [6] }\end{array}$ & $\begin{array}{l}\text { Consider } \\
\text { problem of multi- } \\
\text { view face } \\
\text { detection and } \\
\text { proposed Deep } \\
\text { Dense Face } \\
\text { Detector. That } \\
\text { does not require } \\
\text { pose/landmark } \\
\text { annotation to } \\
\text { detect faces of } \\
\text { orientations } \\
\text { based on deep } \\
\text { CNN. They } \\
\text { analyzed scores } \\
\text { of face detector } \\
\text { for faces in } \\
\text { different } \\
\text { orientations and } \\
\text { found 1) able to } \\
\text { detect faces of } \\
\text { different angles, } \\
\text { 2) a correlation } \\
\text { between } \\
\text { distribution of } \\
\text { positive samples } \\
\text { in the training set } \\
\text { and scores of the } \\
\text { given face } \\
\text { detector. }\end{array}$ & $\begin{array}{l}\text { The method's } \\
\text { performance was } \\
\text { improved using } \\
\text { sampling and } \\
\text { sophisticated } \\
\text { data. Evaluations } \\
\text { on datasets show } \\
\text { that single-model } \\
\text { face detector has } \\
\text { better } \\
\text { performance. }\end{array}$ \\
\hline $\begin{array}{l}\text { Journal of Latex } \\
\text { Class Files 2015, } \\
\text { P. Tzirakis et al } \\
\text { [7] }\end{array}$ & $\begin{array}{lr}\text { An emotion } \\
\text { recognition } \\
\text { system using } \\
\text { auditory and } \\
\text { visual modalities. } \\
\text { To capture } \\
\text { emotions } \\
\text { speaking. A }\end{array}$ & $\begin{array}{l}\text { It outperforms as } \\
\text { compared to the } \\
\text { traditional } \\
\text { approaches based } \\
\text { on auditory and } \\
\text { visual } \\
\text { handcrafted }\end{array}$ \\
\hline
\end{tabular}

\begin{tabular}{|c|c|c|}
\hline & $\begin{array}{l}\text { to extract } \\
\text { features from the } \\
\text { speech, for visual } \\
\text { modality a deep } \\
\text { residual network } \\
\text { proposed to be } \\
\text { insensitive to } \\
\text { outliers to model } \\
\text { the context. So, } \\
\text { Long Short-Term } \\
\text { Memory are } \\
\text { networks are } \\
\text { utilized. System } \\
\text { trained in an end- } \\
\text { to-end fashion } \\
\text { and also taking } \\
\text { advantage of the } \\
\text { correlations. }\end{array}$ & features. \\
\hline $\begin{array}{l}2015 \text { Hindawi, } \\
\text { W. Hu et al [8] }\end{array}$ & $\begin{array}{l}\text { Deep CNN are } \\
\text { employed to } \\
\text { classify hyper- } \\
\text { spectral images. } \\
\text { The architecture } \\
\text { of classifier } \\
\text { contains five } \\
\text { layers with } \\
\text { weights ( input, } \\
\text { convolutional, } \\
\text { max pooling, full } \\
\text { connection, and } \\
\text { output layer. }\end{array}$ & $\begin{array}{l}\text { Results based on } \\
\text { several data sets } \\
\text { show that the } \\
\text { method can } \\
\text { achieve better } \\
\text { performance than } \\
\text { traditional } \\
\text { methods, such as } \\
\text { SVM and } \\
\text { traditional deep } \\
\text { learning- } \\
\text { methods. }\end{array}$ \\
\hline $\begin{array}{l}2015 \text { ACM, Z. } \\
\text { Yu et al [9] }\end{array}$ & $\begin{array}{l}\text { Focus on SFEW } \\
2.0 \text { dataset, seeks } \\
\text { to classify a set } \\
\text { of images into } 7 \\
\text { emotions. It } \\
\text { contains a face } \\
\text { detection } \\
\text { module, followed } \\
\text { by classification } \\
\text { module using } \\
\text { ensemble of } \\
\text { multiple deep } \\
\text { CNN. It is } \\
\text { initialized } \\
\text { randomly and } \\
\text { pre-trained. }\end{array}$ & $\begin{array}{l}\text { First minimizing } \\
\text { the log likelihood } \\
\text { loss, by } \\
\text { minimizing hinge } \\
\text { loss. The method } \\
\text { generates result } \\
\text { on FER dataset. } \\
\text { It also achieves } \\
55.96 \% \text { and } \\
61.29 \% \text { r } \\
\text { respectively. }\end{array}$ \\
\hline $\begin{array}{l}\text { arXiv } 9 \text { May } \\
\text { 2017, X. Yin et } \\
\text { al [10] }\end{array}$ & $\begin{array}{l}\text { They explore } \\
\text { multi-task } \\
\text { learning for face } \\
\text { recognition. A } \\
\text { multi-task CNN } \\
\text { for face } \\
\text { recognition } \\
\text { where identity } \\
\text { classification is }\end{array}$ & $\begin{array}{l}\text { An energy-based } \\
\text { weight analysis } \\
\text { to show CNN- } \\
\text { MTL work. They } \\
\text { observe that the } \\
\text { side tasks serve } \\
\text { as regularizations } \\
\text { to disentangle the } \\
\text { variations from }\end{array}$ \\
\hline
\end{tabular}




\begin{tabular}{|c|c|c|}
\hline & $\begin{array}{l}\text { the main task. } \\
\text { Second, develop } \\
\text { a weighting } \\
\text { scheme to assign } \\
\text { the loss weight. } \\
\text { Third, a pose- } \\
\text { directed multi- } \\
\text { task CNN by } \\
\text { grouping } \\
\text { different poses to } \\
\text { learn features. }\end{array}$ & $\begin{array}{l}\text { the learnt identity } \\
\text { features. } \\
\text { Experiments } \\
\text { demonstrate the } \\
\text { effectiveness of } \\
\text { the approach. } \\
\text { The approach is } \\
\text { also applicable } \\
\text { for pose-invariant } \\
\text { face recognition } \\
\text { and better } \\
\text { performance. }\end{array}$ \\
\hline $\begin{array}{l}\text { Springer Nature } \\
\text { 2017, P. M. M. } \\
\text { Kumar et al [11] }\end{array}$ & $\begin{array}{l}\text { The problems } \\
\text { facing by blind } \\
\text { people fall in the } \\
\text { category of } \\
\text { navigation } \\
\text { through indoor } \\
\text { and outdoor } \\
\text { consisting of } \\
\text { various obstacles } \\
\text { and recognition } \\
\text { of person in front } \\
\text { of them. }\end{array}$ & $\begin{array}{l}\text { Stored images of } \\
\text { friends, relatives } \\
\text { in the database to } \\
\text { recognize a } \\
\text { person in front of } \\
\text { blind user, to } \\
\text { gives voice aid to } \\
\text { user. It is a face } \\
\text { recognition and } \\
\text { navigation } \\
\text { system. It } \\
\text { provides precise } \\
\text { and quick } \\
\text { messages. }\end{array}$ \\
\hline $\begin{array}{l}\text { IEEE 2018, M. } \\
\text { A. et al [12] }\end{array}$ & $\begin{array}{l}\text { That generates a } \\
\text { dataset called the } \\
\text { T-Dataset used to } \\
\text { train the BPNN. } \\
\text { Provides a high } \\
\text { distinction layer } \\
\text { between training } \\
\text { images to } \\
\text { converge faster } \\
\text { and better } \\
\text { accuracy and also } \\
\text { used } \\
\text { histogram tB } \\
\text { prove that. }\end{array}$ & $\begin{array}{l}\text { Achieved higher } \\
\text { recognition } \\
\text { accuracy with } \\
\text { less cost. They } \\
\text { produce a } \\
\text { competitive face } \\
\text { recognition } \\
\text { performance. }\end{array}$ \\
\hline $\begin{array}{l}2016 \text { IEEE, Z. } \\
\text { Wu et al [13] }\end{array}$ & $\begin{array}{l}\text { Thermal face } \\
\text { recognition } \\
\text { depends on the } \\
\text { hand-crafted } \\
\text { features, requires } \\
\text { efforts to select } \\
\text { features and has } \\
\text { lower recognition } \\
\text { rate. }\end{array}$ & $\begin{array}{l}\text { CNN architecture } \\
\text { for TFR. A new } \\
\text { type of ANN can } \\
\text { learn features } \\
\text { from raw data. } \\
\text { Results on face } \\
\text { database show } \\
\text { CNN achieves } \\
\text { higher } \\
\text { recognition rate. }\end{array}$ \\
\hline $\begin{array}{l}2018 \text { IEEE, S. } \\
\text { Kumaar et al [14] }\end{array}$ & $\begin{array}{l}\text { It consists of two } \\
\text { ANN, first CNN } \\
\text { predicts } 20 \text { facial } \\
\text { key-points in } \\
\text { image }\end{array}$ & $\begin{array}{l}\text { The accuracies } \\
\text { are } 67.4 \% \text { and } \\
74.8 \% \text { for } \\
\text { prediction and } \\
\text { classification }\end{array}$ \\
\hline
\end{tabular}

\begin{tabular}{|l|l|l|}
\hline & $\begin{array}{l}\text { second ANN } \\
\text { classifies subject. }\end{array}$ & respectively. \\
\hline $\begin{array}{l}\text { Elsevier 2018, } \\
\text { M. F. Hansen et } \\
\text { al [15] }\end{array}$ & $\begin{array}{l}\text { Non-invasive } \\
\text { biometrics, CNN } \\
\text { model that train } \\
\text { using an accurate pig } \\
\text { artificially } \\
\text { augmented data. }\end{array}$ & $\begin{array}{l}\text { recognition with } \\
\text { rates of 96.7\% on } \\
1553 \text { images. }\end{array}$ \\
\hline
\end{tabular}

Table.1 Review summary

\section{PROPOSED WORK}

The proposed work is motivated to study the multiple pose based face recognition system. A number of highly accurate models for recognizing faces in multiple poses are presented in recent years by different contributors, but most of the techniques either less accurate or computationally cost effective. Therefore the proposed work is focused on exploring the accurate pose based face recognition model. Additionally the proposed model is extended to offer the technique for partial face recognition. Thus we established solution for two limitations of the current available approaches:

1. Recognition of partial faces

2. Recognition of face after small change in face images (i.e. smiling face, crying face)

Basically, even small changes in face can impact on the performance of the learning algorithm for face recognition. Therefore need to be developing a feature selection and classification method by which we achieve the required consequences. Therefore the required model is demonstrated in figure 3.1.

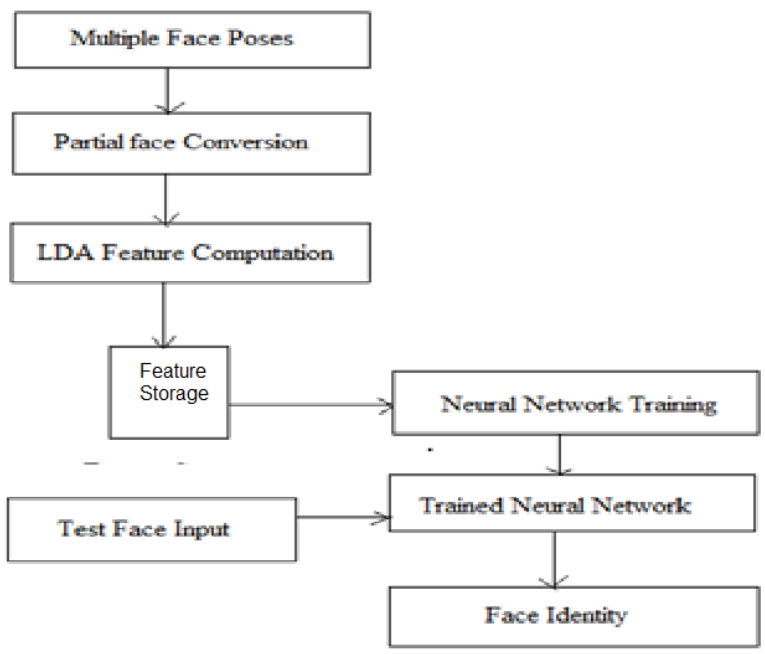

Figure.1 proposed model

The proposed model works in two modules. In first the training is provided to the model and then testing module is provided. Thus to demonstrate the required model ORL dataset is used. In this dataset we consider on 40 classes or identities and for each class we use 10 different poses. Means total of 400 images of 40 
people is used. Among them the $70 \%$ of randomly selected images are used to train the model and $30 \%$ of data is used for testing. The system accepts the input sample images and for partial pose recognition the images are partitioned. These partitioned dataset is used for training and testing of the system.

Further the features from the face images are computed and stored in a database. In order to compute the features the LDA is used. The stored image features in database are learned by the artificial neural network (ANN). After learning the ANN is able to classify the data or faces by producing the test image samples. Finally based on the classification outcomes the performance of the model is computed. The proposed partial face recognition model is used in various other tasks also using multi-pose face recognition technique.

1. Biometric identity based authentication system

2. Identification of faces even some changes on face are found

This section provides the overview of the proposed model which is being developed in further.

\section{CONCLUSION \& FUTURE WORK}

The face recognition is a classical domain of research and development. Therefore a review of existing tools and technology is presented and summarized first. According to the summary of face recognition models we concluded a number of different domains and applications. A significant developments are contributed in this domain i.e. authentication, navigation, and others. But a number of challenges are remaining to fix. In this work we consider the issue of changes in faces to recognize the multiple poses and also able to recognize the identity by partially captured face images. Therefore in this presented work an artificial neural network is employed for recognizing the human partial faces. Those concept usages the LDA based feature extraction to carry out this task. By using this we demonstrate the model for future development and implementation. The proposed model implemented in near future and their performance is measured and reported.

\section{REFERENCES}

[1] D. Bhamare, P. Suryawanshi, "Review on Reliable Pattern Recognition with Machine Learning Techniques”, Fuzzy Information and Engineering, 10:3, 362-377

[2] F. P. Mahdia, Md. M. Habibb, Md. A. R. Ahadb, S. Mckeeverc, A. S. M. Moslehuddinb, P. Vasanta, "Face recognition-based real-time system for surveillance”, Intelligent Decision Technologies 11 (2017) 79-92

[3] A. K. Jain, S. Pankanti, S. Prabhakar, L. Hong, A. Ross, J. L. Wayman, "Biometrics: A Grand Challenge", Proceedings of International Conference on Pattern Recognition, Cambridge, UK, Aug. 2004

[4] M. Hassaballah, S. Aly, "Face recognition: challenges, achievements and future directions”, IET Comput. Vis., 2015, Vol. 9, Iss. 4, pp. 614-626, 614 \& The Institution of Engineering and Technology 2015

[5] M. P. Beham, S. M. M. Roomi, “A Review of Face Recognition Methods”, International Journal of Pattern Recognition and Artificial Intelligence Vol. 27, No. 4 (2013) 1356005 (35 pages)
[6] S. S. Farfade, M. Saberian, L. J. Li, "Multi-view Face Detection Using Deep Convolutional Neural Networks”, ICMR'15, June 2326, 2015, Shanghai, China Copyright is held by the owner/author(s). ACM 978-1-4503-3274-3/15/06

[7] P. Tzirakis, G. Trigeorgis, M. A. Nicolaou, B. Schuller, S. Zafeiriou, "End-to-End Multimodal Emotion Recognition using Deep Neural Networks”, Journal of Latex Class Files, Vol. 14, No. 8, Aug 2015

[8] W. Hu, Y. Huang, L. Wei, F. Zhang, H. Li, “Deep Convolutional Neural Networks for Hyperspectral Image Classification”, Hindawi Publishing Corporation Journal of Sensors Volume 2015, Article ID 258619, 12 pages

[9] Z. Yu, C. Zhang, "Image based Static Facial Expression Recognition with Multiple Deep Network Learning”, ICMI 2015, November 9-13, 2015, Seattle, WA, USA c 2015 ACM ISBN 978$1-4503-3912-4 / 15 / 11$

[10] X. Yin, X. Liu, "Multi-Task Convolutional Neural Network for Pose-Invariant Face Recognition”, arXiv:1702.04710v2 [cs.CV] 9 May 2017

[11] P. M. Kumar, U. Gandhi, R. Varatharajan, G. Manogaran, R. Jidhesh, T. Vadive1, "Intelligent face recognition and navigation system using neural learning for smart security in Internet of Things”, Springer Science+Business Media, LLC, part of Springer Nature 2017

[12] M. A. Abuzneid, A. Mahmood, "Enhanced Human Face Recognition Using LBPH Descriptor, Multi-KNN, and BackPropagation Neural Network", 2169-3536, 2018 IEEE, Vol 6, 2018

[13] Z. Wu, M. Peng, T. Chen, "Thermal Face Recognition Using Convolutional Neural Network", 2016 International Conference on Optoelectronics and Image Processing, 978-1-5090-08803/16/\$31.00 @2016 IEEE

[14] S. Kumaar, R. M. Vishwanath, S. N. Omkar, A. Majeedi, A. Dogra, "Disguised Facial Recognition Using Neural Networks", 2018 IEEE 3rd International Conference on Signal and Image Processing, 978-1-5386-6396-7/18/\$31.00 @2018 IEEE

[15] M. F. Hansen, M. L. Smith, L. N. Smith, M. G. Salter, E. M. Baxter, M. Farish, B. Grieved, "Towards on-farm pig face recognition using convolutional neural networks", Computers in Industry, 2018, 98, 145-152

\section{AUTHOR'S PROFILE:}

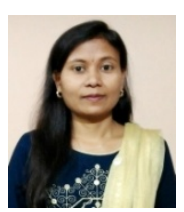

Savita Bairagi received her B.E. / B.Tech. In Information Technology at SGSITS Indore and also perusing M.E. / M.Tech. In Computer Science and Engineering at LNCT, Indore both degrees from the University of R.G.P.V. (Rajiv Gandhi Proudyogiki Vishwavidyalaya) Bhopal, Madhya Pradesh. She is working as a Research Scholar as well as Faculty from Government Polytechnic College, Dhar Madhya Pradesh. She is working in Computer Science Department. She has 04 years of Teaching Experience in Government Polytechnic College (Department of Computer Science), Dhar Madhya Pradesh. As well as 04 Years of School Teaching Experience in Computer Physics, and Mathematics. Her area of interests include: - Computer Networks, 
Neural Network, Ad-hoc Networks, MANET, Network Security, Information Security, Web Security, Big Data, Cryptography, Distributed Systems, Information Security , Data Mining \& Image Processing. She has attended more than 10 Workshops / Seminars in Various events etc.

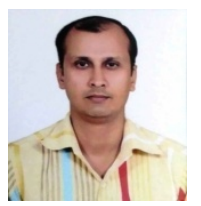

HEMANT KUMAR GUPTA M.Tech is working in LNCTS (RIT), Indore (MP) as Asst. Professor besides HOD in CSE Department with over 12 years of experience. He received Best Faculty Awards 2017 for actively handling the examinations and good academic feedback at LNCTS, Indore Madhya Pradesh. He also worked as software developer with ALM software Khargone for 05 month. He has published 11 papers in International journal, 06 papers in International conferences, 02 papers in National conference and 05 papers in National seminar. His area of main research interest include -: Big data analysis, Data security, Hybrid cryptography, 4G wireless network.

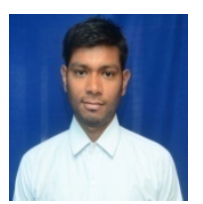

ASHOK PANWAR received has Three Year Polytechnic Diploma in Computer Science and Engineering, B.E. / B. Tech. and M.E. / M. Tech. Degree both in Computer Science and Engineering from the University of R.G.P.V. (Rajiv Gandhi Proudyogiki Vishwavidyalaya) Bhopal, Madhya Pradesh. He is currently working as a Technical Officer (C) in ECIL (Electronics Corporation of India Limited), India, against the site requirements of NPCIL (Nuclear Power Corporation of India Limited), TAPS (Tarapur Atomic Power Station 1 to 4) Tarapur, Mumbai, Maharashtra, India. Working in ACS (Access Control System) and Simulator Department. He is Ex. Worker / Employee in Defence Research \& Development Organisation (DRDO) in Defence Scientific Information \& Documentation Centre (DESIDOC) Lab, Govt. of India, Ministry of Defence, in Department of Knowledge Management
Division (KMD), Metcalfe House, Near Civil Lines, New Delhi, Delhi110054, India. He has One year of Teaching Experience in Computer Networking. His area of Main Research Interests include:- Ad-hoc Networks, MANET, Network Security, Cryptography, Big Data, Information Security, Web Security, Distributed Systems, Document Analysis and Cryptanalysis, Cyber Security, Data Mining \& Image Processing. He has guided 90 above Graduate Students, and 50 above Post Graduate Students. He is Allotted Membership of London Journal Press. He has Published 03 Research Papers in International Journals. He is many PPT's Published in Various topics. He has attended One National Level Conference. He has attended Two National Level Event's of Microsoft Dream Spark Yatra at IET - DAVV, Indore. He has attended Five Day's National Level Workshop on Android Security System. He has attended Two Day's National Level Workshop in NS2 (Network Simulator and Design 2) and attended Three Day's National Level Seminar on Udhyamita Jagrukta Shivir Organized by SADMAP Bhopal. He has attended more than 45 Workshops / Seminars in Various events.

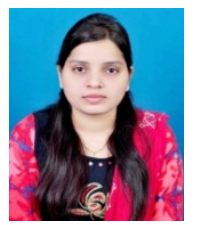

BHAVANA PANWAR received her M.Sc. in Mathematics Science in 2016 and B.Sc. in Computer Science in 2014 both Degree's from the University of DAVV (Devi Ahilya Vishwavidyalay) Indore, Madhya Pradesh, and also Pursuing B. Ed. (Bachelor of Education) from the University of DAVV (Devi Ahilya Vishwavidyalay) Indore, Madhya Pradesh. She is working as a Research Scholar as well as Faculty from Sardar Vallabh Bhai Patel H.S. School Kasrawad, Khargone, Madhya Pradesh. She has 02 years of Teaching Experience in Mathematics as well as Computer. Her area of interests include:- Real and Complex Analysis, Fluid Mechanics, Mathematical and Numerical Analysis, Quantitative Risk Analysis, Computer Networks, Algebra and Cryptography, Big Data, Document Analysis, Network Security, Optimistic, Distributed Systems, Information Security and Data Mining. She is Published 01 Research Paper in International Journal. She has attended more than 20 Workshops / Seminars in Various events etc. 